\title{
Role of intensity fluctuations in nonlinear pulse propagation
}

\author{
B. Crosignani* and C. H. Papas \\ California Institute of Technology, Pasadena, California 91125 \\ P. Di Porto \\ Fondazione Ugo Bordoni, Istituto Superiore P.T., Viale Europa, Roma, Italy \\ Received June 18, 1980

\begin{abstract}
The effect of intensity fluctuations and the finite coherence time of the field on the propagation of nonlinear optical pulses is discussed. In particular, the statistical properties of the carrier are shown to affect the power level for soliton propagation.
\end{abstract}

The transmission of nonlinear optical pulses in dispersive dielectric waveguides was first investigated by Hasegawa and Tappert, ${ }^{1}$ who pointed out the possibility of propagating stable solitary solutions (bright and dark solitons) through nonlinear media using reasonable intensities. This is achieved through compensation between the sharpening effect on the pulse arising from the nonlinear self-induced changes in the refractive index and the broadening effect derived from ordinary dispersion. Later, the influence of the fiber structure was more explicitly considered by Jain and Tzoar,2,3 who demonstrated the possibility of obtaining bright soliton propagation even at normal dispersion by exploiting the transverse inhomogeneity of the medium. The influence of the longitudinal inhomogeneity (that is, along the propagation direction $z$ of the fiber) has been taken into account by Bendow and Gianino. ${ }^{4}$

In the above-mentioned papers, the analytic signal $\mathbf{E}(\mathbf{r}, z, t)$ of the electric field is assumed to be of the form

$$
\mathbf{E}(\mathbf{r}, z, t)=\hat{e} U(\mathbf{r}) \Phi(z, t) e^{i q z-i \omega_{0} t},
$$

where $\hat{e}$ is a unit polarization vector, $U(\mathbf{r})$ contains the radial dependence of the field, and $\Phi(z, t)$ is a slowly varying deterministic function. This last assumption is equivalent to neglecting the effects of the finite coherence time of the field arising from its statistical fluctuations. The most appropriate way of taking these fluctuations into account, namely, looking for the envelope solitons having as a carrier a field possessing general time-coherence properties, is to write $\Phi(z, t)$ in the form

$$
\Phi(z, t)=F(z, t) S(z, t),
$$

where $F(z, t)$ describes the stochastic nature of the field (or, equivalently, of the exciting source) and $S(z, t)$ is the deterministic signal that one wishes to propagate.

In most practical situations, the detector performs an averaging operation of the instantaneous intensity over time intervals much larger than the characteristic fluctuation times of $F(z, t)$ and much shorter than the duration of $S(z, t)$. This operation is consistent with the assumption that the spectrum of the pulse envelope is negligible compared with the carrier spectrum, which also justifies the factorization of $\Phi(z, t)$ into the product of a rapidly fluctuating quantity $F(z, t)$ and of a slowly varying deterministic quantity $S(z, t)$. Accordingly, the measured intensity is proportional to

$$
\left\langle|\Phi(z, t)|^{2}\right\rangle=\left\langle|F(z, t)|^{2}\right\rangle S^{2}(z, t),
$$

where the symbol $\langle\ldots\rangle$ can be assumed to indicate either the time or the ensemble average.

The equation obeyed by the single realization of $\Phi(z, t)$ is given by ${ }^{3}$

$$
\begin{gathered}
{\left[p_{1}^{2}-q^{2}+\partial^{2} / \partial z^{2}+2 i q \partial / \partial z+2 i k_{0} k_{0}{ }^{\prime} \partial / \partial t\right.} \\
-\left(k^{\prime}{ }^{2}+k_{0} k_{0}{ }^{\prime \prime}\right) \partial^{2} / \partial t^{2} \\
\left.+2 \alpha\left(n_{2} / n_{0}\right) k_{0}{ }^{2}|\Phi|^{2}\right] \Phi(z, t)=0,
\end{gathered}
$$

where $p_{1}$ is the propagation constant of the mode excited in the fiber and $\alpha$ is a factor taking into account its transverse spatial configuration, $n_{2}$ is the nonlinear index of refraction, and $n_{0}, k_{0}, k_{0}{ }^{\prime}$, and $k_{0}{ }^{\prime \prime}$ are, respectively, the index of refraction, the wave number in the material, and its first and second derivatives with respect to $\omega$, evaluated at the central frequency $\omega_{0}$ of the field.

If we multiply Eq. (4) by $\Phi^{*}$ and its complex conjugate by $\Phi$, and add and subtract the resulting expressions, we obtain

$$
\begin{gathered}
\left(\Phi^{*} \partial^{2} \Phi / \partial z^{2}-\Phi \partial^{2} \Phi^{*} / \partial z^{2}\right) \\
+2 i q\left(\Phi^{*} \partial \Phi / \partial z+\Phi \partial \Phi * / \partial z\right)+2 i k_{0} k_{0}{ }^{\prime}\left(\Phi^{*} \partial \Phi / \partial t\right. \\
\left.+\Phi \partial \Phi^{*} / \partial t\right)+\left(k_{0}{ }^{2}+k_{0} k_{0}{ }^{\prime \prime}\right)\left(\Phi \partial^{2} \Phi * / \partial t^{2}\right. \\
\left.-\Phi * \partial^{2} \Phi / \partial t^{2}\right)=0
\end{gathered}
$$

and

$$
\begin{aligned}
2\left(p_{1}{ }^{2}-q^{2}\right) \mid & ||^{2}+\Phi^{*} \partial^{2} \Phi / \partial z^{2}+\Phi \partial^{2} \Phi * / \partial z^{2} \\
& +2 i q(\Phi * \partial \Phi / \partial z-\Phi \partial \Phi * / \partial z) \\
+ & 2 i k_{0} k_{0}{ }^{\prime}(\Phi * \partial \Phi / \partial t-\Phi \partial \Phi * / \partial t) \\
-\left(k_{0}{ }^{\prime 2}+k_{0} k_{0}{ }^{\prime \prime}\right)\left(\Phi * \partial^{2} \Phi / \partial t^{2}+\Phi \partial^{2} \Phi * / \partial t^{2}\right) & \\
& +4 \alpha\left(n_{2} / n_{0}\right) k_{0}{ }^{2}|\Phi|^{4}=0 .
\end{aligned}
$$

By time averaging over Eqs. (5) and (6), we are able to write 


$$
q \partial S / \partial z+k_{0} k_{0}{ }^{\prime} \partial S / \partial t=0
$$

which implies that $S(z, t)$ is a function of $\xi=(t-z)$ $\left.v_{g}\right) / \tau$, with

$$
v_{g}=q /\left(k_{0} k_{0}{ }^{\prime}\right)
$$

and $\tau$ the pulse width, and

$$
\begin{aligned}
& {\left[p_{1}{ }^{2}-q^{2}+1 / z_{c}{ }^{2}-\left(k_{0}{ }^{2}+k_{0} k_{0}{ }^{\prime \prime}\right) / t_{c}{ }^{2}\right] S} \\
& +\partial^{2} S / \partial z^{2}-\left(k_{0}{ }^{2}+k_{0} k_{0}{ }^{\prime \prime}\right) \partial^{2} S / \partial t^{2} \\
& +2 \alpha\left(n_{2} / n_{0}\right) k_{0}{ }^{2} \eta\left\langle A^{2}\right\rangle S^{3}=0 .
\end{aligned}
$$

In deriving Eqs. (7) and (9), we have assumed that

$$
F=A e^{i \gamma} \text {, }
$$

where the amplitude $A$ and the phase $\gamma$ are statistically independent stationary variables (in particular, $\left\langle A^{2}(z, t)\right\rangle$ is independent from $z$ and $t$ ), and we have defined

$$
\begin{aligned}
1 / z_{c}{ }^{2} & =\left\langle A \partial^{2} A / \partial z^{2}\right\rangle /\left\langle A^{2}\right\rangle-\left\langle(\partial \gamma / \partial z)^{2}\right\rangle, \\
1 / t_{c}{ }^{2} & =\left\langle A \partial^{2} A / \partial t^{2}\right\rangle /\left\langle A^{2}\right\rangle-\left\langle(\partial \gamma / \partial t)^{2}\right\rangle,
\end{aligned}
$$

and

$$
\eta=\left\langle A^{4}\right\rangle /\left\langle A^{2}\right\rangle^{2} .
$$

Now, by following a procedure analogous to that of Ref. 3, one finds a solution of Eq. (9) for bright solitons (a similar derivation can be given for dark solitons) to be of the form $S(\xi)=S_{0}$ sech $\xi$, with

$$
\begin{aligned}
q^{2}=p_{1}{ }^{2}+\alpha\left(n_{2} / n_{0}\right) \eta k_{0}{ }^{2}\left\langle A^{2}\right\rangle S_{0}{ }^{2}\left(1+\tau^{2} / t_{c}{ }^{2}\right) \\
+1 / z_{c}{ }^{2}-1 /\left(t_{c}{ }^{2} v_{g}{ }^{2}\right)
\end{aligned}
$$

and

$$
\left\langle A^{2}\right\rangle S_{0}^{2}=\left[1 / v_{g}{ }^{2}-\left(k_{0}{ }^{2}+k_{0} k_{0}{ }^{\prime \prime}\right)\right] /\left[\alpha\left(n_{2} / n_{0}\right) \eta k_{0}{ }^{2} \tau^{2}\right] .
$$

It is worthwhile to note that Eqs. (14) and (15), although based on the explicit assumption that $\tau \gg t_{c}$, are as valid for a rigorously monochromatic carrier $\left(t_{c}=\infty, \eta\right.$ $=1$ ), since in this case no averaging operation is necessary because of the absence of fluctuations. In fact, the results of Ref. 3 are immediately recovered by setting $t_{c}=\infty, z_{c}=\infty$, and $\eta=1$.
The main result of the research described in this Letter consists in the appearance of the factor $\eta$ in the denominator of Eq. (15), which decreases the power level required for soliton propagation with respect to the case of an amplitude-stabilized carrier. Thus the power required for employing a single-mode laser $(\eta=1)$ is twice that of a chaotic source $(\eta=2)$. In practice, $\eta$ is always within the range interval $1 \leq \eta \leq 2$, although it can in principle assume any larger value. ${ }^{5}$ The reduction of the power necessary for soliton propagation that is due to intensity fluctuations is an example of a general property according to which the average rate of a nonlinear optical process is affected by statistical fluctuations of the field. 6

This research was supported by the U.S. Air Force Office of Scientific Research under grant no. AFOSR77-3451.

* B. Crosignani is on leave of absence from Fondazione Ugo Bordoni, Roma (Italy).

\section{References}

1. A. Hasegawa and F. Tappert, "Transmission of stationary nonlinear optical pulses in dispersive dielectric fibers. I. Anomalous dispersion," Appl. Phys. Lett. 23, 142-144 (1973); "Transmission of stationary nonlinear optical pulses in dispersive dielectric fibers. II. Normal dispersion,” Appl. Phys. Lett. 23, 171-173 (1973).

2. M. Jain and N. Tzoar, "Propagation of nonlinear optical pulses in inhomogeneous media," J. Appl. Phys. 49, 4649-4654 (1978).

3. M. Jain and N. Tzoar, "Nonlinear pulse propagation in optical fibers," Opt. Lett. 3, 202-204 (1978).

4. B. Bendow and P. D. Gianino, "Theory of nonlinear pulse propagation in inhomogeneous waveguides," Opt. Lett. 4, 164-166 (1979).

5. M. Bertolotti, B. Crosignani, and P. Di Porto, "On the statistics of Gaussian light scattered by a Gaussian medium," J. Phys. A: Gen. Phys. 3, L37-L38 (1970).

6. Y. R. Shen, "Quantum theory of nonlinear optics," in Proceedings of the International School of Physics "Enrico Fermi," Course XLII, R. J. Glauber, ed. (Academic, New York, 1969), pp. 473-492. 